\title{
Electromyographic properties of the myometrium of the pony mare during pregnancy*
}

\author{
G. J. Haluska†, J. E. Loweł and W. B. Currie \\ Department of Animal Science, and $\ddagger$ Department of Clinical Sciences, Cornell University, Ithaca, \\ New York 14853-4801, U.S.A.
}

\begin{abstract}
Summary. Recordings of uterine electrical activity were made from 5 pregnant pony mares from Day 141 to 320 of pregnancy. Three types of activity were identified. Short, medium and long bursts were quantified as the percentage of time each occurred during the hour analysed and further categorized according to frequency, amplitude and duration. The uterus was most active during the early stages recorded and became increasingly quiescent after Day 240 . Short-burst activity was greatest when the uterus was most quiescent. Long bursts showed the greatest percentage of activity until Day 220 and then decreased. Medium-burst activity was present throughout the period studied and high amplitude synchronous medium bursts peaked at Day 221-240.
\end{abstract}

\section{Introduction}

Electromyographic (EMG) activity of the uterus has been recorded from pregnant sheep (Harding et al., 1982; Krishnamurti et al., 1982; Toutaine et al., 1983), pregnant goats (Taverne \& Sheerboom, 1984), pregnant sows (Taverne et al., 1979a), pregnant cows (Zerobin \& Sporri, 1972) and from the non-pregnant cyclic mare (Taverne et al., 1979b). Despite the relatively unusual nature of the hormonal environment present in the mare during pregnancy, no attempt has been made previously to describe the electrical and contractile activity of the myometrium during this stage.

Progesterone and oestradiol-17 $\beta$ fluctuate throughout pregnancy in the mare with progesterone at high concentrations early in pregnancy (Holtan et al., 1975) and oestrogens (Nett et al., 1973), including oestradiol-17 $\beta$ (Haluska, 1985), at very high concentrations early in the second half of pregnancy. The timing of the major increase and decrease in oestradiol-17 $\beta$ in terms of day of pregnancy has also been shown to vary between mares, and the concentration of this hormone seems to be related to photoperiod (Haluska, 1985). Plasma concentrations of relaxin increase and remain elevated throughout the second half of pregnancy (Stewart \& Stabenfeldt, 1981). Relaxin decreases spontaneous uterine activity in other species (Porter, 1972; Schwabe et al., 1978; Porter et al., 1981; Prud'homme et al., 1983). Finally, 13,14-dihydro-15-keto-prostaglandin F-2 $\alpha$ (PGFM) is known to be elevated during the second half of pregnancy in mares (Barnes et al., 1978; Haluska, 1985). These four hormones have been implicated in myometrial function. This report presents EMG data collected from mares during pregnancy and will correlate the EMG data with previously reported changes in the concentrations of the four principal myometrial regulatory hormones. Parts of the results have been reported in abstract form (Haluska et al., 1983).

\footnotetext{
*Reprint requests to Dr W. B. Currie.

$\nmid$ Present address: Oregon Regional Primate Research Center, 505 N.W. 185th Avenue, Beaverton, Oregon 97006, U.S.A.
} 


\section{Materials and Methods}

Animals. Animal care and daily operational procedures were as described by Haluska et al. (1987).

Surgery. Five mares had four sets of bipolar electrodes surgically implanted in the uterus. Surgery was performed on Days 99, 129, 132, 134 and 163 of pregnancy. The mares received a preliminary i.v. anaesthetic of ketamine (Ketaset: Bristol Laboratories, Syracuse, NY) and xylazine (Rompun: Bayvet, Shawnee, KS) and, after an endotracheal tube was inserted, were maintained to effect during surgery with halothane in oxygen. The uterus was exposed by mid-ventral laparotomy and the pregnant horn was located.

Bipolar electrodes, each consisting of two Teflon-coated multistranded stainless-steel wires (AS 633, Cooner Wire Co., Chatsworth, CA), were sutured into the myometrium and the wires of each set were located $5 \mathrm{~mm}$ apart. The electrodes were placed in the pregnant horn near the tubo-uterine junction, above the bifurcation, in the uterine body below the bifurcation, and as close as possible to the cervix. The Teflon coating was removed from $2 \mathrm{~mm}$ of the wire that was embedded in the myometrium. The eight wires, plus an indifferent electrode (free in the peritoneal cavity) were tracked subcutaneously to an area above the flank and exteriorized.

Electromyographic recording. Each electrode set was joined to the appropriate lead off a Grass polygraph. EMG signals were amplified through Grass AC preamplifers (Model 7P5) and the signals were transcribed to the oscillograph at a chart speed of 10 to $100 \mathrm{~mm} / \mathrm{min}$ as circumstance demanded. Signal filters were set at $10 \mathrm{and} 35 \mathrm{~Hz}$. Gain varied between electrodes but was constant for each individual electrode throughout the experiment.

The recording area consisted of two stalls in an isolated area of the barn where there was minimal environmental interference. The stalls were separated by a room which housed the polygraph and where active monitoring of the mares' behaviour was possible without interference.

Electromyographic analysis. To avoid environmental effects interfering with normal EMG signals, all analysis was done from recordings made when the mares were known not to have been disturbed by human presence. Therefore, recordings obtained from 12:00 to 14:00 $\mathrm{h}$ were used for each day examined.

EMG signal analysis consisted of quantification of the duration of voltage burst activity. Individual events were designated as bursts separated by at least $5 \mathrm{sec}$. Burst activity was subdivided into three types: short bursts (trains of single spikes lasting $<7 \mathrm{sec}$ ); medium bursts (trains of single spikes lasting between 7 and $120 \mathrm{sec}$ ); and long bursts (trains of short and medium bursts lasting $>120 \mathrm{sec}$ ). Periods of inactivity were designated as quiet. The results were expressed as the percentage of time each type of activity occurred during the 2-h period analysed as the mean ( \pm s.e.m.) for each type of burst activity.

The recordings were further examined to incorporate analysis of frequency and amplitude of EMG activity. Frequency was expressed as the number of bursts of each type of activity per hour. Amplitude was divided into: low $(<50 \mu \mathrm{V})$; intermediate $(50-100 \mu \mathrm{V})$; and high $(>100 \mu \mathrm{V})$ amplitude bursts and expressed as the number of bursts/h in each category.

Statistics. Statistical significance was determined by one-way analysis of variance for unequal numbers of datum points. Linear contrasts using Sheffe's adaptation were made to determine significance between individual or group means.

\section{Results}

General

Four uterine electrodes were successfully implanted in the myometrium of each of the 5 mares used. One mare, No. 205, spontaneously aborted her fetus on Day 285, 186 days after surgery. Necropsy findings indicated that the fetus and placenta were of normal size and development and had no gross lesions. The abortion was determined to be secondary to bacteraemia by Staphylococcus aureus.

\section{Electromyographic activity}

During this study, every attempt was made to minimize environmental influences, especially human presence in the recording area. An example of myometrial EMG and simultaneous electrocardiographic (ECG) recording (which was used as a measure of sympathetic activity) is shown in Fig. 1. At the first upward arrow, a person entered the stall with the mare and there was an immediate response both in EMG and ECG.

The percentage of time that each type of activity occurred was averaged over all electrode pairs, hours, and mares and divided into 9 stages, each consisting of 20 days, beginning at Day 141 and ending at Day 320. Figure 2(a) shows that the myometrium became increasingly quiescent as pregnancy progressed: percentage quiescent $(Q)$ ranged from $44 \cdot 3 \pm 5 \%$ during the first stage to 


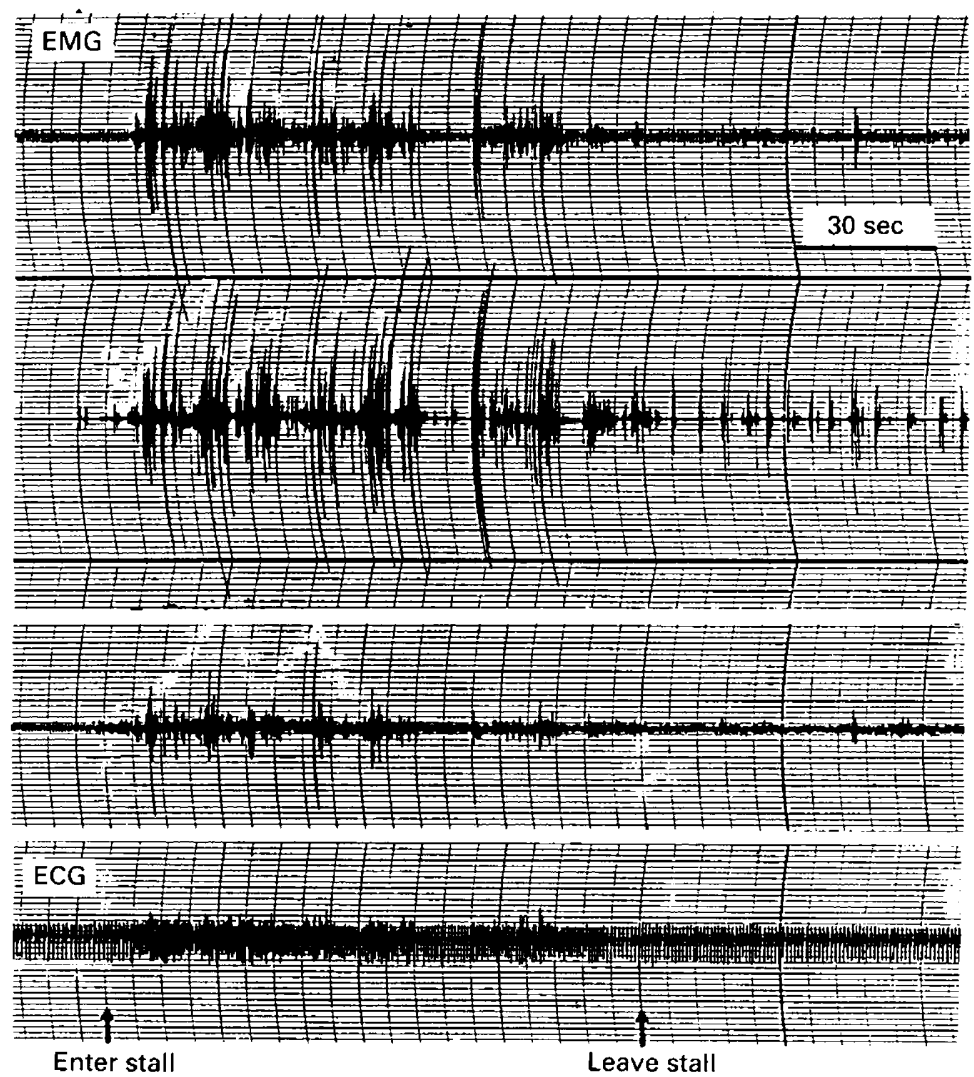

Fig. 1. Myometrial EMG recording and concurrent ECG from one mare at Day 294 of pregnancy. Arrows indicate the points at which a person entered the stall and left the stall.

$69.6 \pm 4.5 \%$ during the final stage of pregnancy. The period encompassing Days 241 to 320 was significantly different $(P<0.001)$ from the 5 stages earlier in pregnancy.

Short-burst (SB) activity increased significantly $(P<0.001)$ from a mean of $3.0 \%$ (Days 140 to 240 ) to $7 \cdot 6 \%$ from Day 241 to Day 300 as shown in Fig. 2(b). There was a significant $(P<0.05)$ positive correlation $(r=0.69)$ between $\%$ Q and \% SB. SB activity was greatest during periods of relative myometrial quiescence, but was considered to be of little, if any, physiological significance.

Medium-burst (MB) activity for the stages between Days 141-160, 201-220, 221-240 and 281-300 were significantly $(P<0.001)$ greater than all other stages of pregnancy. During the final stage (Days 301-320), there was a striking decrease $(P<0.001)$ in MB activity to $17.6 \pm 2.5 \%$ as seen in Fig. 2(c).

Long-burst (LB) activity was at its highest level during the early stages analysed, as shown in Fig. 2(d). There was a significant $(P<0.001)$ decrease in LB activity from about $18 \%$ in early pregnancy to $<2 \%$ during the final stage of pregnancy.

\section{Frequency of EMG activity}

The frequencies of MB and LB activities were significantly $(P<0.01)$ and positively correlated with their respective \% activities (MB, $r=0.78$; LB, $r=0.96$ ) (Fig. 3). The frequency of MB activity was greatest during Days $221-240(68 \cdot 1 \pm 7 \cdot 1$ bursts $/ \mathrm{h})$, and Days 281-300 (60.0 \pm 8.4 bursts $/ h$ ). The lowest frequency of MB activity occurred during the last stage of pregnancy 

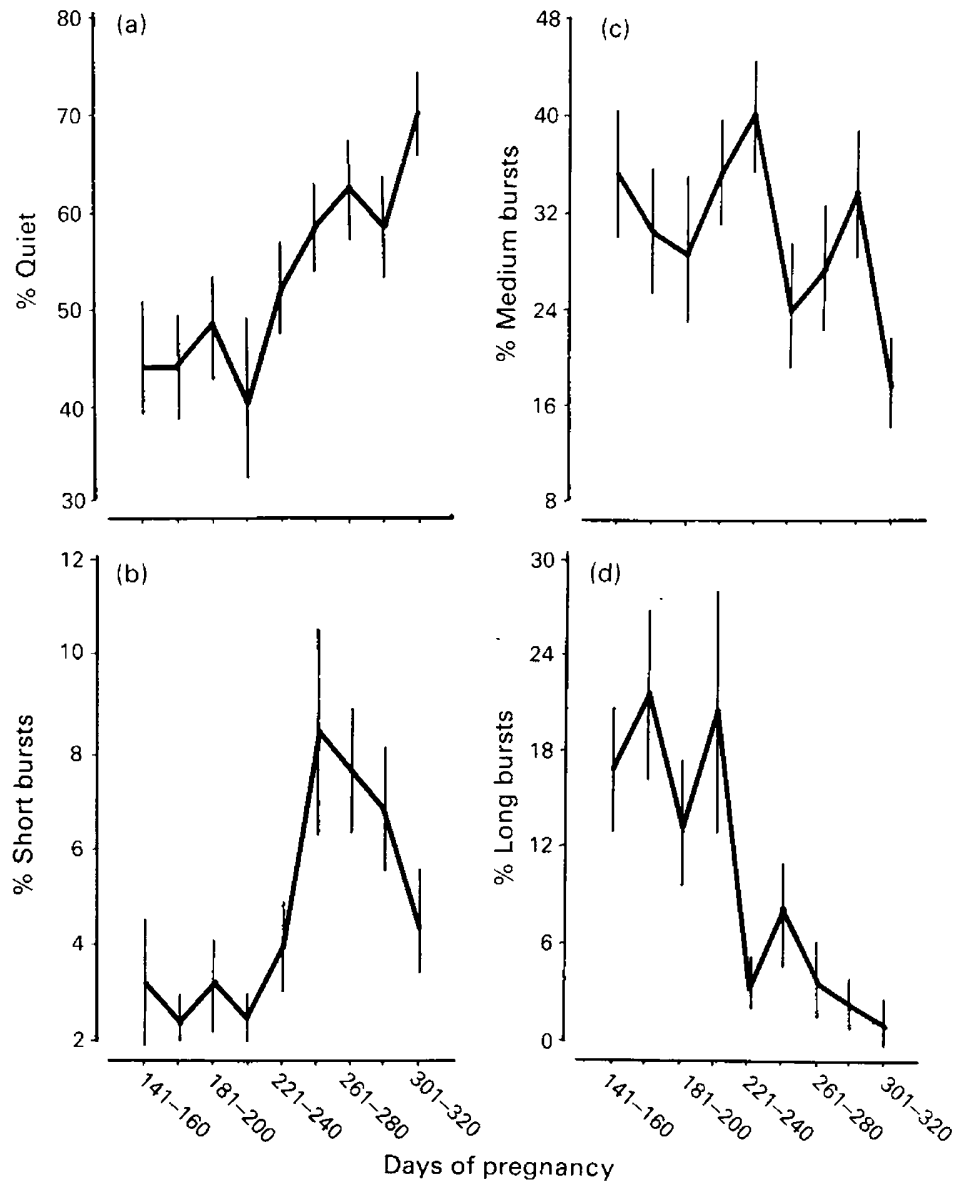

Fig. 2. Mean ( \pm s.e.m.) percentage of time that the pregnant myometrium showed (a) no activity, (b) short burst activity, (c) medium burst activity and (d) long burst activity. Results were collected from 5 mares and the days analysed were grouped into stages as shown on the abscissa. Note that the scale on the ordinate is different in each graph.

$(34 \cdot 1 \pm 4 \cdot 6$ bursts $/ \mathrm{h})$. The frequency of LB activity fell to $0 \cdot 4 \pm 0 \cdot 2$ bursts/h in the final stage of pregnancy, after being maintained at 3 bursts/h until Days 201-220.

\section{Amplitude of EMG activity}

LB activity was predominantly of intermediate or high amplitude (Fig. 4) and the frequency mimicked the general pattern of change shown in Fig. 2(d). Medium bursts (MB), which are known to predominate during the perinatal period in other species, changed such that the frequency of high-amplitude MB activity was greatest during the period between Days 221 and 280. Even though the frequency of low- and intermediate-amplitude $\mathrm{MB}$ followed the general pattern of total MB frequency, the frequency of high amplitude bursts diverged from the general pattern between Days 221 and 280 .

\section{Duration of EMG activity}

The duration of MB activity decreased significantly $(P<0.005)$ as pregnancy progressed. The longest duration occurred during Days 181-200 (31.4 $\pm 3.9 \mathrm{sec})$, it decreased to $22 \cdot 1 \pm 1 \cdot 1 \mathrm{sec}$ at Days 221-240, and the shortest duration occurred during the final stage of pregnancy 


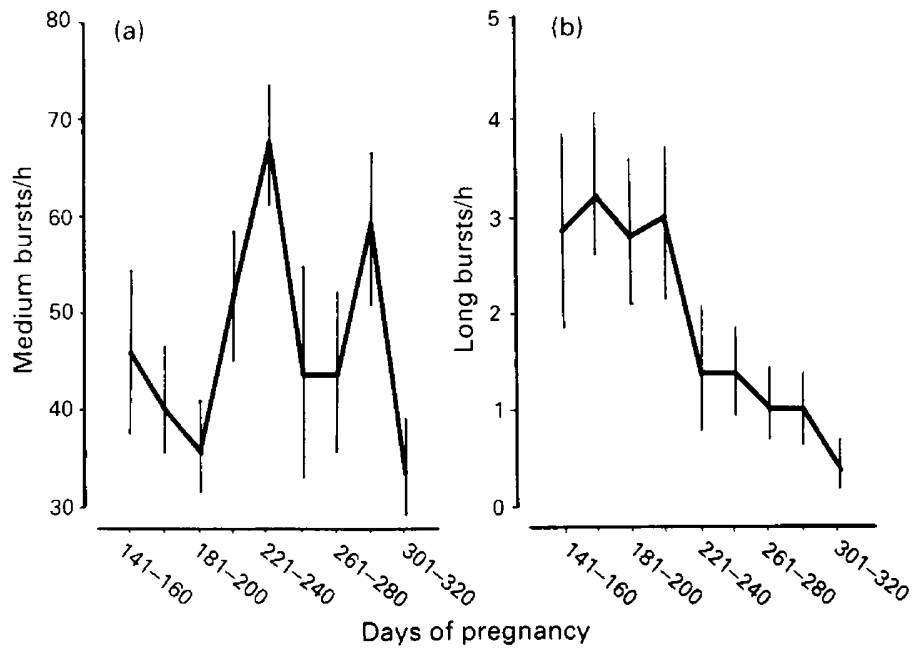

Fig. 3. Mean ( \pm s.e.m.) frequency of uterine EMG bursts in 5 pregnant mares: (a) frequency of medium bursts, (b) frequency of long bursts. Note that the scale on the ordinate is different in each graph.

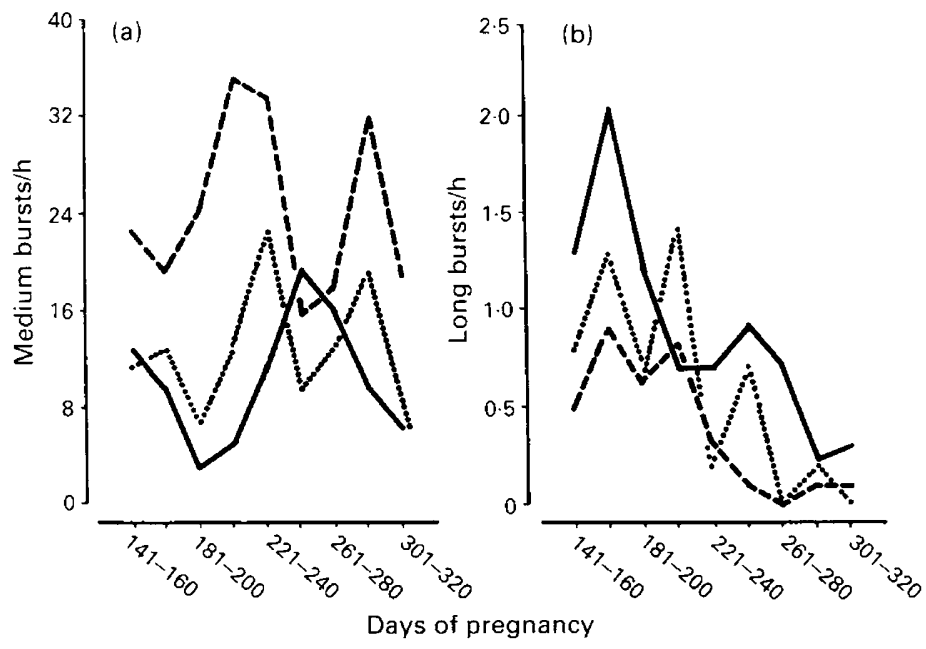

Fig. 4. Mean amplitude of uterine EMG bursts from 5 pregnant mares. The results are expressed as the frequency of low-amplitude bursts $(<50 \mu \mathrm{V},----)$, intermediate-amplitude bursts $(50-100 \mu \mathrm{V}), \cdots \cdots$, and high-amplitude bursts ( $>100 \mu \mathrm{V}, \longrightarrow$ ): (a) medium bursts, (b) long bursts. Note that the scale on the ordinate is different in each graph.

$(15.9 \pm 0.9 \mathrm{sec})$. Duration of LB activity also showed a progressive decrease that was significant at $P<0.05$. The longest bursts occurred in the first stage of pregnancy examined $(271.8 \pm 53.6 \mathrm{sec})$, compared to bursts lasting $158 \cdot 6 \pm 19 \cdot 5 \mathrm{sec}$ in the final stages of pregnancy.

\section{Discussion}

The unusual steroid endocrinology of the pregnant mare was the stimulus for this study. The drastic changes in the relative plasma concentrations of oestradiol to progesterone seen at midpregnancy in the mare (a change comparable to that normally seen at parturition in other species), 
suggested that the mare may be a good model for studying the control of uterine function during pregnancy, and also raised many questions of managerial and clinical significance for the horse.

Chronically implanted bipolar electrodes as used here may be prone to impedance changes which could bias long-term trends in amplitude of potentials. The primary analysis was based on percentage of time that each category of bursts occupied, the parameter least likely to be influenced by possible changes in electrode characteristics. Our observations are confined to once daily 2-h periods of analysis, intentionally chosen to minimize environmental artefacts as indicated by the recordings in Fig. 1. This precludes any consideration of possible diurnal changes in activity; some indications of a circadian rhythm in electromyographic activity were apparent during the last days before parturition (Haluska et al., 1987).

One of the most important observations of this study is that the myometrium of the pregnant mare becomes increasingly more quiescent as pregnancy progresses. In mid-pregnancy the myometrium is electrically active $66 \%$ of the time while in late pregnancy it is active only $30 \%$ of the time. Since progesterone is at its lowest values and the oestrogens are at their highest concentrations during the last third of pregnancy, some additional factor could be responsible for the control of myometrial activity which, in many species, is attributed mainly to the sustained influence of progesterone.

A likely factor for this function is the peptide hormone, relaxin. Relaxin has been shown to inhibit spontaneous myometrial activity in the rat, mouse, hamster, human (Schwabe et al., 1978), guinea-pig (Porter, 1972), and sheep (Porter et al., 1981; Prud'homme et al., 1983). Relaxin also mimics the effects of progesterone in maintaining distensibility of the uterus (Currie, 1979), presumably by affecting the connective tissue components of the uterus. Furthermore, relaxin and progesterone, which independently are ineffective, together inhibit contraction amplitude of human myometrial strips in vitro (Beck et al., 1982). Relaxin is produced by the horse placenta (Stewart \& Stabenfeldt, 1982). In pregnant mares, peripheral plasma concentrations of relaxin increase significantly between Days 76 and 100 from undetectable values and thereafter remain elevated until parturition (Stewart \& Stabenfeldt, 1981). Plasma concentrations of relaxin increase as those of progesterone decrease from the high levels of early pregnancy; oestrogens, also of placental origin, are increasing at the same time. If the effects of relaxin on the myometrium are the same in the mare as in the species noted above, a suppression of spontaneous activity would be expected, leading to a more quiescent uterus. If relaxin does maintain distensibility of the uterus, the mechanical consequences of any remaining electrical events would be dampened.

The tendency towards decreased excitability during the second half of pregnancy in the pony mares is confounded by increased concentrations of both oestradiol-17 $\beta$ and increased prostaglandin (PG) F-2 $\alpha$, as measured by PGFM values (Barnes et al., 1978; Haluska, 1985). PGF-2 $\alpha$ increases the excitability of myometrium and this prostanoid is present at very high levels at parturition in mares (Barnes et al., 1978; Haluska, 1985). Despite this, PGF-2 $\alpha$ seems to have little effect on the myometrium until term. The lack of effect of PGF- $2 \alpha$ is also evident from the failure of exogenous PGs in provoking delivery until term (Leadon et al., 1982). The increase in progesterone during late pregnancy in mares (Holtan et al., 1975; Haluska, 1985), added to the inhibitory effect of relaxin, may prevent the endogenous PGF- $2 \alpha$ from having an excitatory effect.

The striking degree of similarity between \% activity and frequency in all types of electromyographic activity indicates that changes in \% activity are due primarily to changes in frequency of bursts. At Days 200-240, the frequency of medium burst activity reached its highest level during pregnancy, while the duration of these bursts decreased. The frequency of high-amplitude medium bursts was greatest at this time and qualitative evaluation showed the activity to be synchronized over all electrode sites. Thus, when the oestradiol-17\%:progesterone ratio changes in favour of oestradiol in mid-pregnancy, the predominant type of activity of the myometrium is high-amplitude, high frequency bursts lasting about $20 \mathrm{sec}$, a profile characteristic of parturition in many species.

The changes in EMG activity described above are similar to the changes obtained by Csapo \& Takeda (1965) in rabbits exposed to progesterone withdrawal. In all cases, EMG activity changed after withdrawal of progesterone, from low-amplitude long bursts of activity which generated 
negligible intrauterine pressure, to synchronous, high-amplitude shorter bursts $(15-20 \mathrm{sec})$ which generated $20-25 \mathrm{mmHg}$ of intrauterine pressure. Csapo \& Takeda (1965) also noted that the rate of rise of pressure was more rapid with shorter bursts. This indicates that the smooth muscle cells of the myometrium are better electro-coupled and are working as a syncytium rather than in a nonpropagating, asynchronous fashion, as characterized by long trains of low amplitude spike activity.

Taverne et al. (1979b) observed that EMG activity in the cyclic mare seems to be related to the peripheral concentrations of progesterone, with little apparent correlation with the concentration of oestrogen. It would seem that this situation also exists in the pregnant mare, since EMG activity for individual mares shows similar trends throughout pregnancy. The average plasma concentration of progesterone decreases to reach consistently low levels by Day 200 . However, the changes in oestradiol-17 $\beta$ concentrations for individual mares are not consistent with day of pregnancy (Haluska, 1985). As pregnancy progresses there is a significant decrease in all EMG activity despite the fact that oestradiol- $17 \beta$ is present in the peripheral circulation, and at extraordinarily high concentrations in some of the mares. Since there is every indication that the myometrium responds to the changes in oestradiol:progesterone ratio, we suggest that relaxin plays a critical role during the second half of pregnancy to inhibit spontaneous mechanical activity as required for the maintenance of pregnancy in mares.

This work was done in partial fulfilment of the requirements for the Ph.D. degree in the Field of Animal Science at Cornell University (G.J.H.). We thank Frank J. Michel for technical assistance and Suzanne Bremmer for preparation of the manuscript.

\section{References}

Barnes, R.J., Comline, R.S., Jeficott, L.B., Mitchell, M.D., Rossdale, P.D. \& Silver, M. (1978) Foetal and maternal plasma concentrations of 13,14-dihydro-15oxo-prostaglandin $\mathrm{F}$ in the mare during late pregnancy and at parturition. J. Endocr. 78, 201-215.

Beck, P., Szlachter, N., Goldsmith, L.T., Steinetz, B.G. \& Weiss, G. (1982) Synergistic effect of human relaxin and progesterone in human myometrial contractions. Int. J. Gynecol. Obstet. 20, 141-144.

Csapo, A.I. \& Takeda, H. (1965) Effect of progesterone on the electric activity and intrauterine pressure of prepartum and parturient rabbits. Am. J. Obstet. Gynecol. 91, 221-231.

Currie, W.B. (1979) Uterine excitability and distensibility influenced by treatment in vitro with progesterone. Anim. Reprod. Sci. 2, 225-238.

Haluska, G.J. (1985) Electromyographic analysis of the myometrium of the mare correlated with the endocrinology of pregnancy, parturition and the postpartum period. Ph.D. thesis, Cornell University, Ithaca.

Haluska, G.J., Lowe, J.E. \& Currie, W.B. (1983) Electromyographic (EMG) analysis of the equine myometrium during pregnancy. Biol. Reprod. 28, Suppl. 1, 121, Abstr. 178.

Haluska, G.J., Lowe, J.E. \& Currie, W.B. (1987) Electromyographic properties of the myometrium correlated with the endocrinology of the pre-partum and postpartum periods and parturition in pony mares. $J$. Reprod. Fert., Suppl. 35, 553-564.

Harding, R., Poore, E.R., Bailey, A., Thorburn, G.D., Jansen, C.A.M. \& Nathanielsz, P.W. (1982) Electromyographic activity of the non-pregnant and pregnant sheep uterus. Am. J. Obstet. Gynecol. 142, 448-457.
Holtan, D.W., Nett, T.M. \& Estergreen, V.L. (1975) Plasma progestins in pregnant, postpartum and cycling mares. J. Anim. Sci. 40, 251-260.

Krishnamurti, C.R., Kitts, D.D., Kitts, W.D. \& Tompkins, J.G. (1982) Myo-electrical changes in the uterus of the sheep around parturition. J. Reprod. Fert. 64, 59-67.

Leadon, D.P., Rossdale, P.D., Jeffcott, L.B. \& Allen, W.R. (1982) A comparison of agents for inducing parturition in mares in the pre-viable and premature periods of gestation. J. Reprod. Fert., Suppl. 32, 597-602.

Nett, T.M., Holtan, D.W. \& Estergreen, V.L. (1973) Plasma estrogens in pregnant and post partum mares. J. Anim. Sci. 37, 962-970.

Porter, D.G. (1972) Myometrium of the pregnant guinea pig: the probable importance of relaxin. Biol. Reprod. $7,458-464$.

Porter, D.G., Lye, S.J., Bradshaw, J.M.C. \& Kendall, J.Z. (1981) Relaxin inhibits myometrial activity in the ovariectomized non-pregnant ewe. J. Reprod. Fert. 61, 409-414.

Prud'homme, M-J., Martinet, N. \& Picaper, G. (1983) The effect of relaxin on in vivo uterine electromyographic activity in the conscious ovariectomized ewe is oestrogen dependent. Reprod. Nutr. Dévelop. 23, 493-499.

Schwabe, C., Steinetz, B., Weiss, G., Segaloff, A., McDonald, J.K., O'Brian, E. \& Hochman, J. (1978) Relaxin. Recent Prog. Horm. Res. 34, 123-198.

Stewart, D.R. \& Stabenfeldt, G.H. (1981) Relaxin activity in the pregnant mare. Biol. Reprod. 25, 281-289. 
Stewart, D.R. \& Stabenfeldt, G.H. (1982) Determination of the source of equine relaxin. Biol. Reprod. 27, $17-24$.

Taverne, M.A.M. \& Sheerboom, J.E.M. (1984) Myometrial electrical activity during pregnancy and parturition in the pigmy goat. Proc. 10th Int. Congr. Anim. Reprod. \& A.I., Urbana-Champaign, Vol. I, p. 113.

Taverne, M.A.M., Naaktgeboren, C., Elsaesser, F., Forsling, M.L., van der Weyden, G.C., Ellendorff, F, \& Smidt, D. (1979a) Myometrial electrical activity and plasma concentrations of progesterone, estrogen, and oxytocin during late pregnancy and parturition in the miniature pig. Biol. Reprod. 21, 1125-1134.
Taverne, M.AM., van der Weyden, G.C., Fontijne, P., Dieleman, S.J., Pashen, R.L. \& Allen, W.R. (1979b) In-vivo myometrial electrical activity in the cyclic mare. J. Reprod. Fert. 56, 521-532.

Toutaine, P.L., Garcia-Villar, R., Hanzen, C. \& Ruckebusch, Y. (1983) Electrical and mechanical activity of the cervix in the ewe during pregnancy and parturition. J. Reprod. Fert. 68, 195-204.

Zerobin, K. \& Sporri, H. (1972) Motility of the bovine and porcine uterus and fallopian tube. Adv. Vet. Sci. comp. Med. 16, 303-354.

Received 17 March 1987 